\title{
Effect of a Small Amount of Thermoplastic Starch Blend on the Mechanical Recycling of Conventional Plastics
}

\author{
Dan Åkesson ${ }^{1}$ (1) - Gauthaman Kuzhanthaivelu ${ }^{1} \cdot$ Martin Bohlén ${ }^{2}$
}

Accepted: 14 October 2020 / Published online: 24 October 2020

(c) The Author(s) 2020

\begin{abstract}
The usage of bioplastics could increase in the future which may cause contamination of the waste streams of conventional plastics. The objective of this study was to investigate if a small amount of biopolymer contaminating conventional polymers would significantly affect mechanical and thermal properties. A starch-based plastic was first compounded by blending plasticised starch with PLA (polylactic acid). This polymer blend was subsequently compounded with HDPE (high density polyethylene), PP (polypropylene) or PET (polyethylene terephthalate) at $0 \%, 1 \%$ and $5 \%$ of the biopolymer. The compounds were characterised by tensile tests, Charpy impact tests, DSC (differential scanning calorimetry) and FESEM (field emission scanning electron microscopy). Tests showed that PE and PP were not significantly affected in terms of tensile strength and modulus but the elongation at break showed a strong reduction. PET was, on the other hand, incompatible with the starchbased plastic. Already at $1 \%$ contamination, PET had lost most of its impact strength.
\end{abstract}

Keywords Bioplastics $\cdot$ Contamination $\cdot$ Mechanical recycling

\section{Background}

Mechanical recycling is often regarded as the preferred method of recycling thermoplastics [1]. As most thermoplastics are not blendable, the most critical step for successful mechanical recycling is the sorting. Considering that there are many different thermoplastics on the market and that these plastics also occur in different colours and with or without different fillers, sorting is obviously rather complicated. A potential problem is that new plastics emerge on the market which evidently further complicates the sorting.

Biopolymers are very attractive as they can be both renewable and biodegradable and have been studied quite intensively over the years. Biopolymers such as polyhydroxyalkanoates (PHA), thermoplastic starch (TPS) and polylactic acid (PLA) are already on the market although currently used in small volumes.

Dan Åkesson

dan.akesson@hb.se

1 Swedish Centre of Resource Recovery, University of Borås, 50190 Borås, Sweden

2 Rise Research Institutes of Sweden, Molndal, Sweden
However, the introduction of bioplastics on the market is not unproblematic. While mechanical recycling for plastics such as high-density polyethylene (HDPE) and polyethylene terephthalate (PET) is already established, bioplastics that are biodegradable may have different properties than petrobased polymers and if volumes increase this could further complicate the sorting process and disturb current recycling regimes [2]. Even if advanced separation techniques are deployed, some plastics will inevitably be missorted and biodegradable plastics may end up in the waste streams of recycled plastics. These plastics may be sensitive to thermal and hydrolytic degradation but the knowledge of how such contamination affects thermal and mechanical properties is hard to predict and this is not well studied. It has been shown that PET is sensitive to contamination and that small amounts of contaminants may lead to cloudiness [3]. Furthermore, while plastics such as HDPE and polypropylene (PP) are not dried before processing, many biopolymers are hygroscopic and need to be carefully dried before melt processing. Thus, if HDPE and PP are contaminated with biopolymers, the humidity could possibly lead to degradation of the biopolymer and to outgassing in an extruder.

The effect of small amounts of biopolymers contaminating conventional polymers is not well studied. Gere and Czigany studied the influence of PLA contaminating recycled 
polyethylene films [4]. It was concluded that $2 \%$ contamination of PLA gave tolerable properties. La Mantia et al. studied the effect of PLA contaminating PET bottle recycling [5]. Tests were done with up to 5\% PLA contamination. Tests showed that the contamination significantly changed the rheological properties. Inspecting the morphology with SEM, a biphasic blend was found, showing that PET and PLA are not blendable. However, the mechanical properties were largely unaffected.

In this study, three conventional polymers were chosenHDPE, PP and PET - and blended with small amounts of a blend of TPS and PLA (TPS/PLA). While starch is an attractive biopolymer that is renewable, biodegradable, abundant and cheap, it is not a thermoplastic polymer. A thermoplastic-like material can be created by plasticising starch. This has been evaluated in a number of studies [6-8]. However, plasticised starch has some drawbacks. As starch is plasticised, the mechanical properties are simultaneously reduced. For that reason, starch is often blended with other polymers which may improve mechanical properties as well as water sensitivity. A wide range of different starch-based polymers have been evaluated [9-13]. In this study, a starchbased polymer was first prepared by blending TPS with PLA using polyethylene glycol (PEG) as a compatibiliser [14]. The biopolymer obtained was then compounded with the conventional polymers to simulate contamination.

\section{Experimental}

\section{Materials}

HDPE (GA7760) and PP (HP648T) were obtained from LyondellBasell. PET (A06 700) was obtained from DSM. Potato starch was obtained from a local grocery store. PLA (3051D) was obtained from NatureWorks. Glycerol (99\%) was obtained from Acros Organics and polyethylene glycol 4000 (PEG 4000, BioUltra), was obtained from Sigma Aldrich.

\section{Processing}

TPS and TPS/PLA were prepared as outlined by Ferrarezi et al., using the same type of microcompounder [14]. Shortly thereafter, $40 \mathrm{wt} \%$ dry glycerol was added to starch. This blend is denoted "TPS". The TPS obtained was then compounded together with PLA and PEG using a twin-screw microcompounder from DSM Xplore. The obtained polymer blend had a composition of $56 \mathrm{wt} \%$ PLA, $19 \mathrm{wt} \%$ TPS and 25 wt\% PEG. This blend is denoted "TPS/PLA".

Test bodies for mechanical testing were produced using the abovementioned instrument. The residence time maintained was two minutes, $70 \mathrm{rpm}$ with a processing temperature of $170{ }^{\circ} \mathrm{C}, 220{ }^{\circ} \mathrm{C}$, and $270{ }^{\circ} \mathrm{C}$ for PE, PP and PET, respectively. The plastic melt was then transferred to a laboratory injection moulder, also from DSM Xplore. The mould temperatures maintained for PE, PP and PET were $40{ }^{\circ} \mathrm{C}, 26{ }^{\circ} \mathrm{C}$ and $130{ }^{\circ} \mathrm{C}$, respectively. Test bodies were produced from the polymer pellet blends for three different concentrations of TPS/PLA as follows: $0 \mathrm{wt} \%, 1 \mathrm{wt} \%$, and $5 \mathrm{wt} \%$. PET pellets were dried overnight at $120{ }^{\circ} \mathrm{C}$ before processing. The blends were denoted with a figure indicating the percentage, for example, HDPE_TPS/PLA1 consists of $1 \mathrm{wt} \%$ TPS/PLA and $99 \mathrm{wt} \%$ HDPE.

\section{Conditioning}

If bioplastics are stored in a humid climate, they may absorb humidity which may lead to polymer degradation when processed. In order to study this, the polymer blends with 0 $\mathrm{wt} \%, 1 \mathrm{wt} \%$ and $5 \mathrm{wt} \%$ were exposed to humidity by placing the pellets in a climate chamber (TK 120, Nüve, Turkey) for $72 \mathrm{~h}$ with $85 \%$ relative humidity at $25{ }^{\circ} \mathrm{C}$. After the conditioning, the polymer blends were blended in a microcompounder and moulded in an injection moulding machine for dog-bone samples, as described above.

\section{Characterisation}

The polymer samples were characterised by tensile tests according to ISO 527. A tensile tester from Tinius Olsen equipped with a load cell of $250 \mathrm{~N}$ (for PE and PP) and $5000 \mathrm{~N}$ (for PET) was employed. The tensile strength was determined using a test speed of $30 \mathrm{~mm} / \mathrm{min}$ and the tensile modulus were determined using a test speed of $1 \mathrm{~mm} / \mathrm{min}$. A minimum of five samples were tested.

Charpy impact tests were done using a QC-639L instrument from Comtech Testing Machines Co. Ltd. (Taiwan). Samples were tested edgewise. Notched HDPE test bodies were tested using a pendulum of $1 \mathrm{~J}$. The samples were notched $0.5 \mathrm{~mm}$ by a QC-640 V-shape Notcher. PP and PET were tested unnotched using a pendulum of $5 \mathrm{~J}$.

Scanning electron microscopy (SEM) was done by an external company using a SIGMA VP-ZEISS equipped with EDS-EBSD-Mapping-Line Scanning from Oxford Instrument Co. Cryo-cuts were made from the tensile dogbone test bodies and the micrographs were taken from the cryo-cut surfaces.

The thermal properties of the samples of different mass sealed in aluminium pans were analysed by dynamical scans using DSC-Q2000 (TA Instruments, USA). The samples were heated from 0 to $200{ }^{\circ} \mathrm{C}$ (HDPE and PP) and $0{ }^{\circ} \mathrm{C}$ to $300^{\circ} \mathrm{C}(\mathrm{PET})$ in the presence of nitrogen at a heating rate of $10^{\circ} \mathrm{C} / \mathrm{min}$. Glass transition temperature $\left(\mathrm{T}_{\mathrm{g}}\right)$, the peak of the melting $\left(\mathrm{T}_{\mathrm{M}}\right)$, cold crystallisation enthalpy $(\Delta \mathrm{Hc})$, and melting enthalpy $\left(\Delta \mathrm{H}_{\mathrm{M}}\right)$ were recorded in the second heating. 
Table 1 Results from the tensile testing

\begin{tabular}{llll}
\hline Polymer & $\begin{array}{l}\text { Tensile } \\
\text { strength } \\
(\mathrm{MPa})\end{array}$ & $\begin{array}{l}\text { Tensile } \\
\text { modulus } \\
(\mathrm{GPa})\end{array}$ & Elongation $(\%)$ \\
\hline HDPE & $28.5(0.9)$ & $1.3(0.06)$ & $1319(308)$ \\
HDPE_TPS/PLA1 & $27.4(0.8)$ & $1.3(0.2)$ & $237.7(168)$ \\
HDPE_TPS/PLA5 & $28.4(1.2)$ & $1.2(0.07)$ & $150.6(56.8)$ \\
PP & $39.6(1.1)$ & $1.7(0.04)$ & $897.9(137)$ \\
PP_TPS/PLA1 & $35.9(1.7)$ & $1.7(0.06)$ & $645.9(36)$ \\
PP_TPS/PLA5 & $34.6(2.5)$ & $1.7(0.06)$ & $280.6(219)$ \\
PET & $66.0(2.8)$ & $2.7(0.6)$ & $22.1(6.9)$ \\
PET_TPS/PLA1 & $65.0(3.9)$ & $2.6(0.6)$ & $6.1(0.7)$ \\
PET_TPS/PLA5 & $26.9(4.5)$ & $2.8(0.9)$ & $2.3(1.1)$ \\
\hline
\end{tabular}

For each individual blend, samples were analysed and the values averaged, and the standard deviation was calculated.

\section{Results}

In the first part of the study, biopolymers contaminating conventional polymers were simulated by compounding small amounts of biopolymers together with the conventional polymers. The results of the mechanical testing are summarised in Table 1. HDPE is relatively unaffected by the addition of the biopolymer. There was no significant change in tensile strength and modulus. However, a clear reduction of the elongation could be seen. A possible explanation is that TPS/PLA was not fully blended in the HDPE matrix. Thus, "islands" of the biopolymer will cause an indication of fracture during mechanical testing (see further the discussion about the SEM micrographs). Gere and Czigany studied the addition of PLA to a mixed film waste consisting of HDPE and low density polyethylene (LDPE) [4]. When 2\% of PLA was added, no significant changes in the tensile properties were recorded. At 10\% contamination of PLA, the elongation was clearly reduced.

As for PP, the tensile strength was reduced from 39.6 to 34.6 MPA. PP has a higher melting point than HDPE. This may cause some polymer degradation of the biopolymer. However, PP is also relatively unaffected by a $5 \%$ contamination. In addition, for PP, a clear reduction in elongation could be seen. As expected, PET was clearly more sensitive to contamination than the two polyolefins. At $5 \%$ contamination, the tensile strength was reduced from 66.0 to $26.9 \mathrm{MPa}$. La Mantia et al. studied small amounts of PLA blended into PET. When 5\% PLA was added, only a very small difference in tensile strength was detected [5]. A fundamental difference is that two different polyesters were blended in that study while in this study PET and a starch-based polymer were blended. As PET contains the reactive ester bond in its backbone, it can react with the hydroxy groups in glycerol and starch. The strong reduction of the tensile strength is therefore expected.

To put these results in context, the contamination of conventional petroleum-based plastics by other petroleumbased plastics has previously been studied. Hope, Bonner and Mile studied the contamination of polyethylenes [15]. When HDPE was contaminated with 5\% PET, there was a clear reduction of the elongation from 490 to $160 \%$. The results of this study roughly confirm the abovementioned study results in the sense that a strong reduction of the elongation was observed.

The Charpy impact strength is shown in Table 2. HDPE is unaffected by the addition of TPS/PLA. For PP, already at $1 \%$ contamination, a significant reduction of the Charpy impact strength can clearly be observed. For PP $+5 \%$ TPS/ PLA, the impact strength was reduced to less than half of its initial value. For PET, the trend is even clearer-with 5\% TPS/PLA, the impact strength was reduced from 63 to 3. As a matter of fact, in terms of impact strength, PET is already sensitive to contamination at $1 \%$ contamination. PET, being a polyester, is sensitive to degradation.

The morphology of the polymer blends was examined by FESEM and the micrographs are shown in Fig. 1. The micrographs were taken from the cryo-cut surfaces. It is obvious that TPS/PLA is not blendable in the conventional polymers and a biphasic morphology can be seen. In HDPE, the biopolymer formed droplets of a few microns in diameter. The biphasic morphology is less distinct for PET with $5 \%$ TPS/PLA. It is possible that the biopolymer degrades quickly at the higher processing temperature of PET, and that hydroxy groups in the biopolymers may have hydrolysed PET which may change the morphology of the polymer blend. In general, the biopolymer seems to be well distributed. As expected, poor adhesion can be seen, with crevices between the biopolymer and the conventional polymer.

Table 2 Notched Charpy impact results for HDPE and unnotched Charpy impact results for PP and PET

\begin{tabular}{ll}
\hline Polymer & $\begin{array}{l}\text { Charpy impact } \\
\text { strength }(\mathrm{kJ} / \\
\left.\mathrm{m}^{2}\right)\end{array}$ \\
\hline HDPE & $3.2(0.5)$ \\
HDPE_TPS/PLA1 & $3.3(0.6)$ \\
HDPE_TPS/PLA5 & $3.3(0.5)$ \\
PP & $56.0(21.1)$ \\
PP_TPS/PLA1 & $33.5(8.5)$ \\
PP_TPS/PLA5 & $20.7(3.8)$ \\
PET & $62.8(29.8)$ \\
PET_TPS/PLA1 & $11.6(2.6)$ \\
PET_TPS/PLA5 & $3.3(0.9)$ \\
\hline
\end{tabular}


(a)

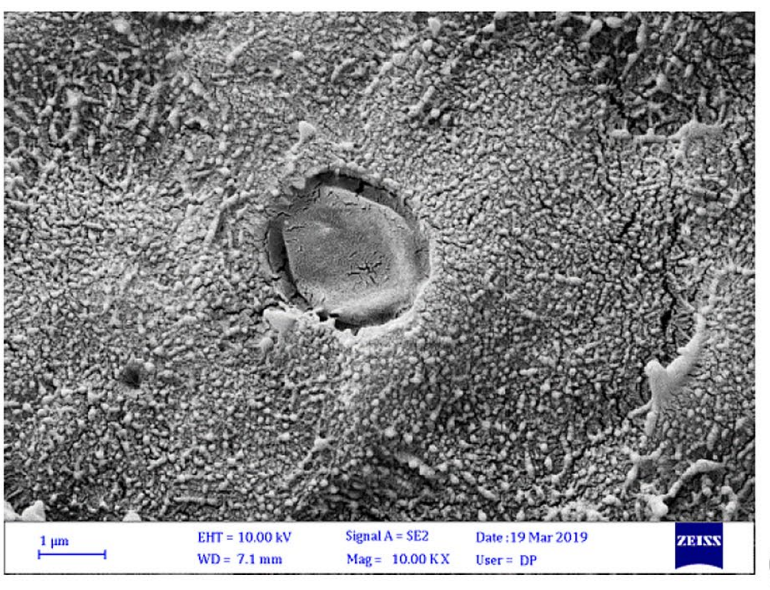

(b)
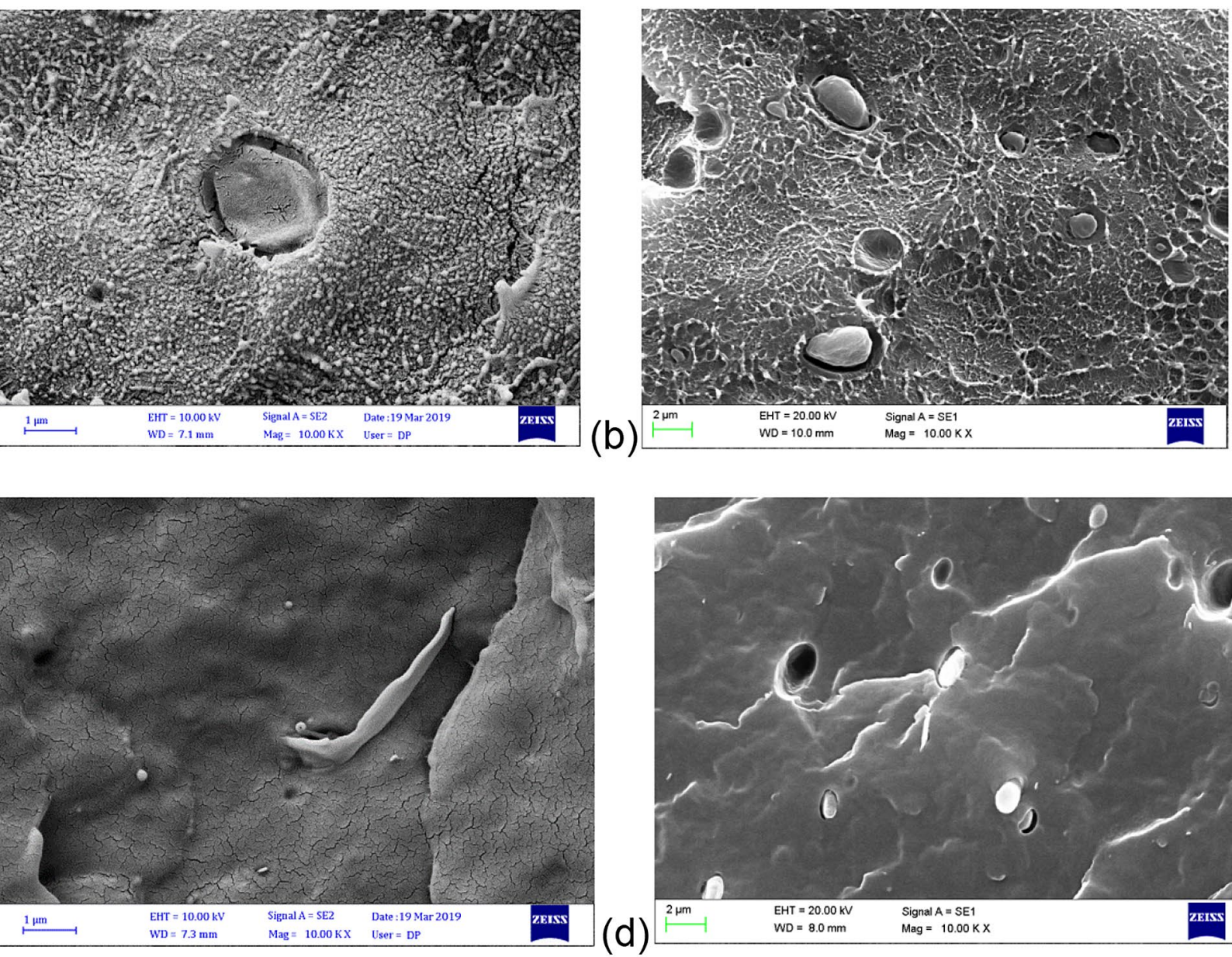

(c)

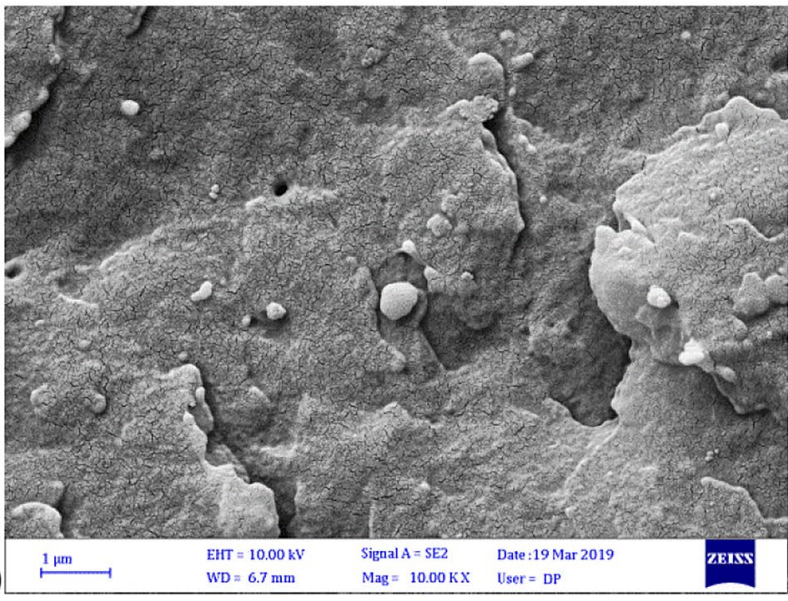

(d)
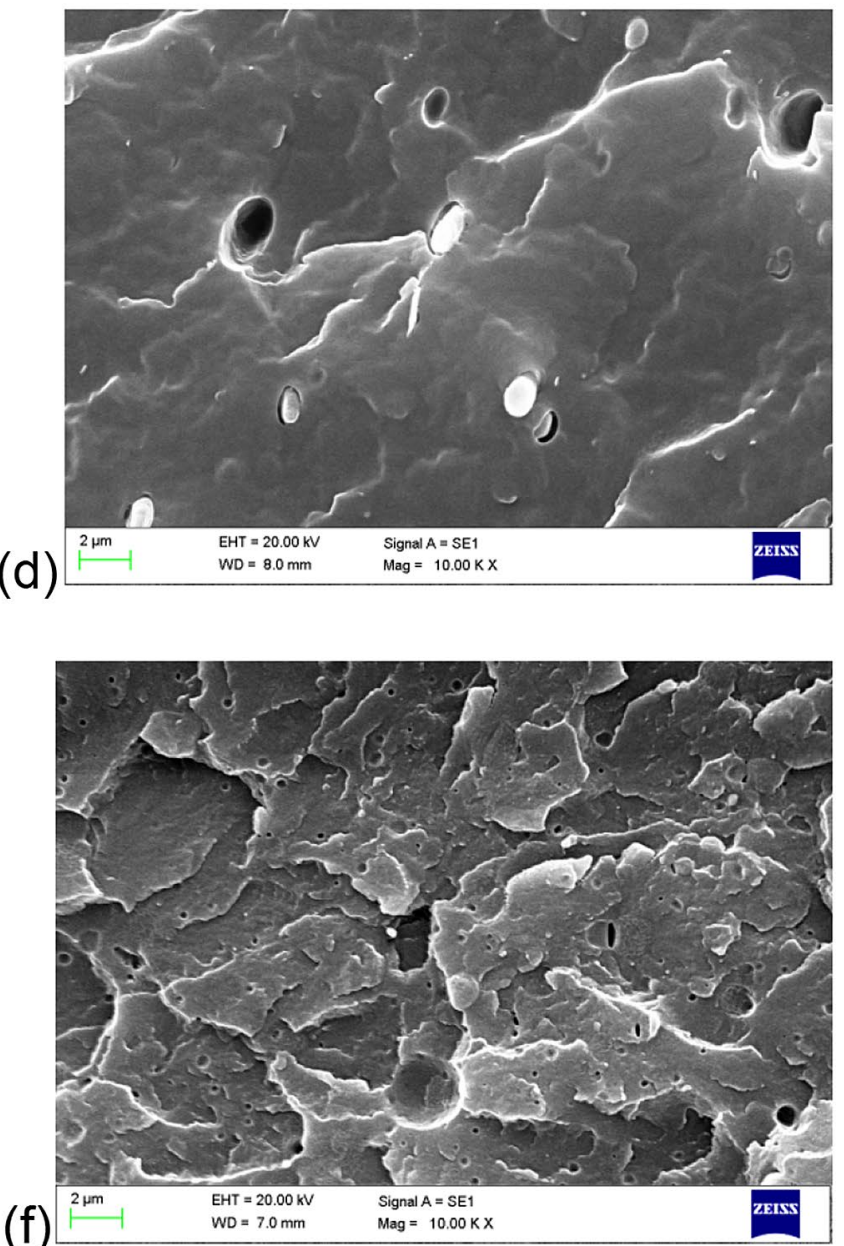

Fig. 1 SEM micrograph for a HPDE with $1 \%$ TPS, b HPDE with 5\% TPS, $\mathbf{c}$ PP with $1 \%$ TPS, $\mathbf{d}$ PP with 5\% TPS, e PET with $1 \%$ TPS, and $\mathbf{f}$ PET with $5 \%$ TPS

The thermal properties of the blends were evaluated by DSC. The curves for HDPE are shown in Fig. 2 and the rest of the result is shown in Table 3. As HDPE is contaminated by the biopolymer, the crystallinity is clearly reduced. Already at $1 \%$ contamination the heat of fusion is reduced from 226 to $80 \mathrm{~J} / \mathrm{g}$, and at $5 \%$ contamination the heat of fusion is further reduced to $69 \mathrm{~J} / \mathrm{g}$. PP basically follows the same pattern, with a strong reduction in crystallinity. For PET, a reduction in the Tg could also be detected as the $\mathrm{Tg}$ was reduced from 83.7 to $71.5^{\circ} \mathrm{C}$. 


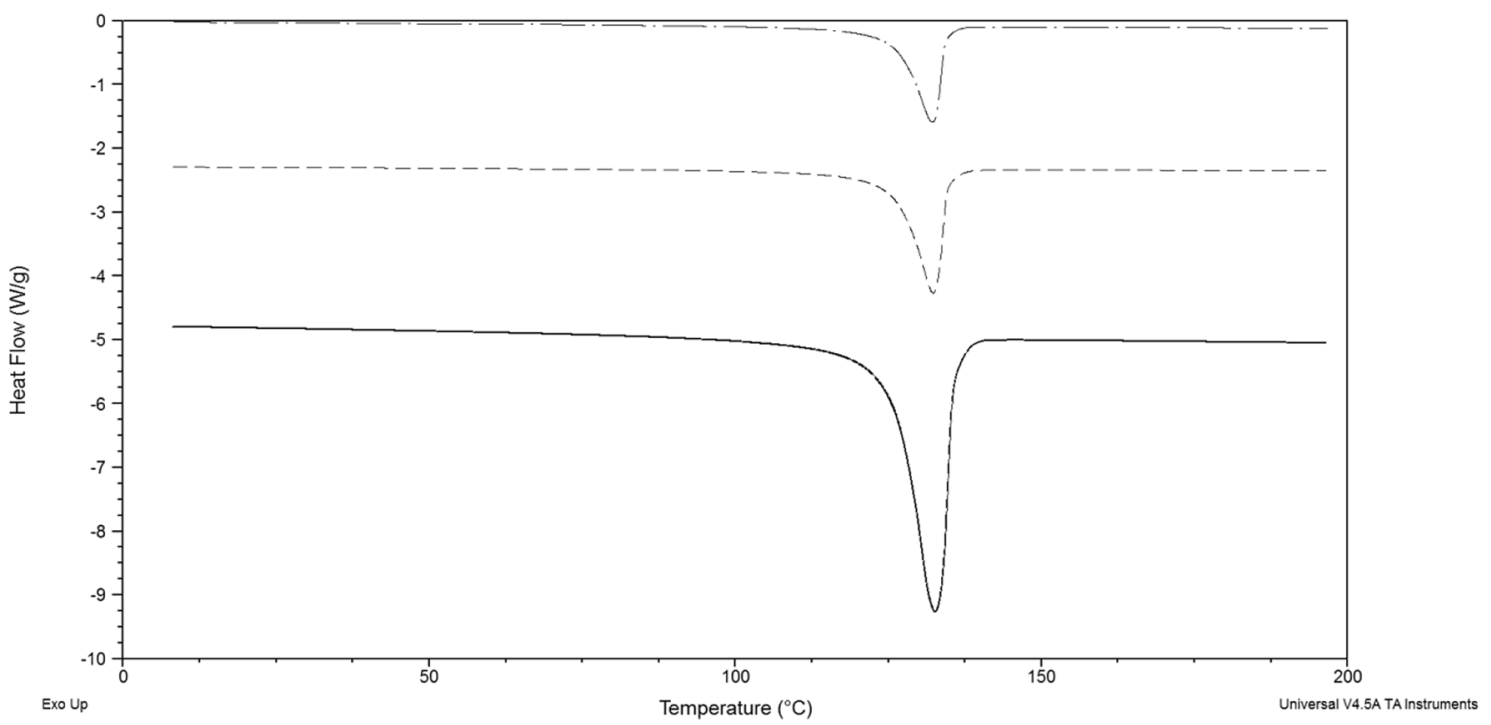

Fig. 2 Characterisation by DSC of neat HDPE (bottom), HDPE_TPS/PLA1 (middle) and HDPE_TPS/PLA5 (top)

Table 3 The results of the thermal characterisation by DSC

\begin{tabular}{lllll}
\hline Polymer & $\Delta \mathrm{H}_{\mathrm{m}}$ & $\mathrm{X}_{\mathrm{c}}$ & $\mathrm{T}_{\mathrm{m}}$ & $\mathrm{T}_{\mathrm{g}}$ \\
\hline HDPE & $225.92(10.3)$ & $77.1(3.5)$ & $132.7(0.0)-$ \\
$\begin{array}{l}\text { HDPE_TPS/ } \\
\quad \text { PLA1 }\end{array}$ & $80.3(6.8)$ & $27.4(2.3)$ & $132.5(0.3)-$ \\
HDPE_TPS/ & $69.0(10.4)$ & $23.6(3.6)$ & $132.1(0.1)-$ \\
$\quad$ PLA5 & & & & \\
PP & $94.0(4.1)$ & $45.4(2.0)$ & $165.1(2.6)-$ \\
PP_TPS/PLA1 & $34.9(2.7)$ & $16.8(1.3)$ & $162.1(0.4)-$ \\
PP_TPS/PLA5 & $34.5(0.8)$ & $16.7(0.4)$ & $161.5(0.2)-$ \\
PET & $31.1(6.9)$ & $22.2(4.9)$ & $254.6(2.3)$ & $83.7(0.7)$ \\
PET_TPS/PLA1 & $14.5(3.2)$ & $10.5(2.3)$ & $254.4(0.1)$ & $81.6(0.8)$ \\
PET_TPS/PLA5 & $20.8(2.3)$ & $15.0(1.7)$ & $251.6(0.5)$ & $71.5(0.0)$ \\
\hline
\end{tabular}

This indicates a strong polymer degradation in line with the tensile test.

\section{Conditioned Samples}

Polyolefins are not hygroscopic, and HDPE and PP are therefore not dried before processing. Possibly, when polyolefins are contaminated by biopolymers, the biopolymer might absorb humidity which could accelerate the degradation of the biopolymer. Consequently, to evaluate this, HDPE and PP contaminated by TPS/PLA were conditioned in a humid environment before processing. PET was not included in this study since it is always dried before processing. The tensile properties are shown in Table 4. This test reveals small differences when comparing samples with and without conditioning. This shows that there is no major polymer degradation caused by the humidity. However, for both tensile strength and modulus, a slight increase can be seen. It is well known that the mechanical properties of TPS can vary with humidity. Soest and Knooren prepared TPS from starch, water and glycerol [6]. The compounded materials were conditioned in a climate chamber at $61 \% \mathrm{RH}$ for 60 days. A clear trend of increasing tensile strength and modulus as a function of the storage time was seen. This is in line with the results of this study.

As can be seen in Table 5, a certain reduction of the crystallinity can be observed. For example, HPDE_TPS/ PLA5 had a crystallinity of $23.6 \%$ but when the contaminated samples were conditioned in a humid climate before processing, the crystallinity was reduced to $15.3 \%$. PP followed the same trend.
Table 4 Tensile properties for sample with and without conditioning

\begin{tabular}{llll}
\hline Polymer & $\begin{array}{l}\text { Tensile strength } \\
\text { (MPa) }\end{array}$ & Elongation (\%) & Modulus (GPa) \\
\hline HDPE/TPS5 (without conditioning) & $28.4(1.2)$ & $10.5(0.3)$ & $1.2(0.1)$ \\
HDPE/TPS5 (with conditioning) & $31.4(0.6)$ & $10.5(0.9)$ & $1.5(0.1)$ \\
PP/TPS5 (without conditioning) & $34.6(2.5)$ & $10.9(0.9)$ & $1.7(0.1)$ \\
PP/TPS5 (with conditioning) & $38.0(1.2)$ & $10.4(0.5)$ & $2.1(0.3)$ \\
\hline
\end{tabular}


Table 5 Characterisation by DSC with and without conditioning of the sample in a humid environment

\begin{tabular}{llll}
\hline Polymer & $\Delta \mathrm{H}_{\mathrm{m}}(\mathrm{J} / \mathrm{mol})$ & $\mathrm{X}_{\mathrm{c}}(\%)$ & $\mathrm{T}_{\mathrm{m}}\left({ }^{\circ} \mathrm{C}\right)$ \\
\hline $\begin{array}{l}\text { HDPE/TPS5 (without condi- } \\
\text { tioning) }\end{array}$ & $69.0(10.4)$ & $23.6(3.6)$ & $132.1(0.1)$ \\
$\begin{array}{l}\text { HDPE/TPS5 (with condition- } \\
\text { ing) }\end{array}$ & $44.7(6.9)$ & $15.3(2.3)$ & $132.3(1.5)$ \\
$\begin{array}{l}\text { PP/TPS5 (without condition- } \\
\text { ing) }\end{array}$ & $34.5(0.8)$ & $16.7(0.4)$ & $161.5(0.2)$ \\
$\begin{array}{l}\text { PP/TPS5 (with conditioning) } \\
\text { PP }\end{array}$ & $21.6(5.0)$ & $10.5(2.4)$ & $161.2(0.2)$
\end{tabular}

In summary, tests where the blends were conditioned in a humid climate before processing reveal that some parameters such as the crystallinity are affected, but under the chosen test conditions, the changes are relatively small.

\section{Conclusions}

The contamination of conventional polymers by biopolymers was studied. Conventional polymers were compounded together with small levels of a biopolymer. The results can be summarised as follows:

- Polyethylene is the most common thermoplastic and it is commonly recycled. Under the chosen experimental conditions, the tensile strength and modulus were relatively unaffected. However, the elongation was significantly reduced. Adding 5\% TPS/PLA to HDPE reduced the elongation from about $1300 \%$ to $150 \%$.

- PP was somewhat more sensitive than HDPE and this could be seen especially for the Charpy impact strength. Adding 5\% TPS/PLA to PP, the Charpy impact strength was reduced from roughly 56 to $21 \mathrm{~kJ} / \mathrm{m}^{2}$.

- As expected, PET was the most sensitive polymer. With polyester groups in its backbone, it is sensitive to degradation. TPS contains hydroxyl groups that can react with PET when the blend is processed at elevated temperatures. This will degrade PET, resulting in clearly inferior mechanical properties and the Charpy impact strength was clearly reduced already at $1 \%$ contamination. We can conclude that TPS/PLA is incompatible with PET.

To what extent may the introduction of biopolymers on the market threaten the mechanical recycling of conventional polymers? This is a hard question to answer since there are many different plastics on the market, both petro-based and bio-based, and more tests are needed than those presented in this article. It is, however, clear, with the chosen experimental set-up in this study, that a small contamination of the biopolymer may cause a reduction of tensile and thermal properties. For HDPE and PP, this is reflected mainly in a reduction of the elongation at break and the Charpy impact strength. However, one should keep in mind that when conventional polymers are recycled, they are also contaminated by other conventional polymers which can lead to a strong reduction of elongation and impact strength as well [15]. The results in this article do not imply that the contamination of biopolymer will lead to an extra-large reduction of thermal and mechanical properties simply because the polymer is biodegradable.

Acknowledgements This study was funded by Stiftelsen AForsk.

Funding Open access funding provided by University of Boras.

Open Access This article is licensed under a Creative Commons Attribution 4.0 International License, which permits use, sharing, adaptation, distribution and reproduction in any medium or format, as long as you give appropriate credit to the original author(s) and the source, provide a link to the Creative Commons licence, and indicate if changes were made. The images or other third party material in this article are included in the article's Creative Commons licence, unless indicated otherwise in a credit line to the material. If material is not included in the article's Creative Commons licence and your intended use is not permitted by statutory regulation or exceeds the permitted use, you will need to obtain permission directly from the copyright holder. To view a copy of this licence, visit http://creativecommons.org/licenses/by/4.0/.

\section{References}

1. Hamad K et al (2013) Recycling of waste from polymer materials: an overview of the recent works. Polym Degrad Stabil 98(12):2801-2812

2. Alaerts L et al (2018) Impact of bio-based plastics on current recycling of plastics. Sustainability 10(5):1487

3. Itim B, Philip M (2015) Effect of multiple extrusions and influence of PP contamination on the thermal characteristics of bottle grade recycled PET. Polym Degrad Stabil 117:84-89

4. Gere D, Czigany T (2018) Rheological and mechanical properties of recycled polyethylene films contaminated by biopolymer. Waste Manag 76:190-198

5. La Mantia FP et al (2012) Effect of small amounts of poly(lactic acid) on the recycling of poly(ethylene terephthalate) bottles. Polym Degrad Stabil 97(1):21-24

6. Van Soest JJG, Knooren N (1997) Influence of glycerol and water content on the structure and properties of extruded starch plastic sheets during aging. J Appl Polym Sci 64(7):1411-1422

7. Róz ALD et al (2006) The effect of plasticizers on thermoplastic starch compositions obtained by melt processing. Carbohydr Polym 63(3):417-424

8. Shi R et al (2007) Characterization of citric acid/glycerol co-plasticized thermoplastic starch prepared by melt blending. Carbohydr Polym 69(4):748-755

9. Ferri JM et al (2018) Poly(lactic acid) formulations with improved toughness by physical blending with thermoplastic starch. J Appl Polym Sci 135(4):45751

10. Li G, Favis Basil D (2010) Morphology development and interfacial interactions in polycaprolactone/thermoplastic-starch blends. Macromol Chem Phys 211(3):321-333

11. Zhou J et al (2009) Preparation and characterization of surface crosslinked TPS/PVA blend films. Carbohydr Polym 76(4):632-638

12. Sabetzadeh M et al (2017) Morphology and rheological properties of compatibilized low-density polyethylene/linear low-density polyethylene/thermoplastic starch blends. J Appl Polym Sci 134(16):44719 
13. Martins AB, Santana RMC (2016) Effect of carboxylic acids as compatibilizer agent on mechanical properties of thermoplastic starch and polypropylene blends. Carbohydr Polym 135:79-85

14. Ferrarezi MMF et al (2013) Poly(ethylene glycol) as a compatibilizer for poly(lactic acid)/thermoplastic starch blends. J Polym Environ 21(1):151-159

15. Hope PS et al (1994) Study of compatibilisers for recovering the mechanical properties of recycled polyethylenes. Plast Rubber Compos process Appl 22:147-158
Publisher's Note Springer Nature remains neutral with regard to jurisdictional claims in published maps and institutional affiliations. 